\title{
Weighted estimates of higher order commutators generated by BMO-functions and the fractional integral operator on Morrey spaces
}

Takeshi lida*

\section{${ }^{*}$ Correspondence:}

tiida@fukushima-nct.ac.jp Department of General Education, National Institute of Technology, Fukushima College, Fukushima, 970-8034, Japan

\begin{abstract}
The purpose of this paper is to investigate the weighted estimates of commutators generated by BMO-functions and the fractional integral operator on Morrey spaces. The main result generalizes the Sawano, Sugano, and Tanaka result to a weighted setting.
\end{abstract}

MSC: $26 \mathrm{~A} 33 ; 42 \mathrm{~B} 25 ; 42 \mathrm{~B} 35$

Keywords: commutator; fractional integral operator; Morrey space; BMO space; weight

\section{Introduction}

The aim of this paper is to investigate the weighted inequalities of commutators generated by BMO-functions and the fractional integral operator on Morrey spaces. The main results particularly is related to [1] and [2]. The authors introduced the condition of weights in [1]. Under a certain condition of the weights, we investigate the weighted estimates of commutators generated by $\mathrm{BMO}$-functions and the fractional integral operator on Morrey spaces. The results recover the inequality in [2].

For $1<p<\infty$, we define $p^{\prime}:=\frac{p}{p-1}$. In this paper, a symbol $C$ is a positive constant. Whenever we evaluate the operator, the constant $C$ may be change from one constant to another. Let $|E|$ denote the Lebesgue measure of $E$. Let $\mathcal{D}\left(\mathbb{R}^{n}\right)$ be the collection of all dyadic cubes on $\mathbb{R}^{n}$. All cubes are assumed to have their sides parallel to the coordinate axes. For a cube $Q \subset \mathbb{R}^{n}$, we use $l(Q)$ to denote the side-length $l(Q)$ and $c Q$ to denote the cube with the same center as $Q$ but with side-length $c l(Q)$. The integral average of a measurable function $f$ over $Q$ is written

$$
m_{Q}(f)=f_{Q} f(x) d x=\frac{1}{|Q|} \int_{Q} f(x) d x .
$$

By a 'weight' we will mean a non-negative function $w$ that is positive measure a.e. on $\mathbb{R}^{n}$. Given a weight $w$ and a measurable set $E$, let

$$
w(E):=\int_{E} w(x) d x .
$$

(c) 2016 lida. This article is distributed under the terms of the Creative Commons Attribution 4.0 International License (http://creativecommons.org/licenses/by/4.0/), which permits unrestricted use, distribution, and reproduction in any medium, provided you give appropriate credit to the original author(s) and the source, provide a link to the Creative Commons license, and indicate if changes were made. 
First we define the Morrey spaces.

Definition 1 Let $1<p \leq p_{0}<\infty$. We define the Morrey space $\mathcal{M}_{p}^{p_{0}}\left(\mathbb{R}^{n}\right)$ by

$$
\mathcal{M}_{p}^{p_{0}}\left(\mathbb{R}^{n}\right):=\left\{f \in L_{\mathrm{loc}}^{p}\left(\mathbb{R}^{n}\right) ;\|f\|_{\mathcal{M}_{p}^{p_{0}}}<\infty\right\}
$$

where for all measurable functions $f$, we define

$$
\|f\|_{\mathcal{M}_{p}^{p_{0}}}:=\sup _{Q \in \mathcal{D}\left(\mathbb{R}^{n}\right)}|Q|^{\frac{1}{p_{0}}}\left(f_{Q}|f(x)|^{p} d x\right)^{\frac{1}{p}} .
$$

\section{Remark 1}

(a) The ordinary Morrey norm is equivalent to the Morrey norm in this paper (see [1]):

$$
\sup _{\substack{Q \subset \mathbb{R}^{n}, Q: \text { cubes }}}|Q|^{\frac{1}{p_{0}}}\left(f_{Q}|f(x)|^{p} d x\right)^{\frac{1}{p}} \cong\|f\|_{\mathcal{M}_{p}^{p_{0}}\left(\mathbb{R}^{n}\right)} .
$$

(b) Hölder's inequality gives us the following inequality: If $1<p \leq q \leq p_{0}<\infty$, then we have

$$
\|f\|_{\mathcal{M}_{p}^{p_{0}}} \leq\|f\|_{\mathcal{M}_{q}^{p_{0}}}
$$

We define the BMO space (see $[3,4])$ as follows.

Definition 2 For an $L_{\text {loc }}^{1}\left(\mathbb{R}^{n}\right)$-function $b$, define

$$
\|b\|_{\mathrm{BMO}}:=\sup _{Q \subset \mathbb{R}^{n}} f_{Q}\left|b(x)-m_{Q}(b)\right| d x,
$$

where the supremum is taken over all cubes $Q \subset \mathbb{R}^{n}$. Define

$$
\operatorname{BMO}\left(\mathbb{R}^{n}\right):=\left\{b \in L_{\text {loc }}^{1}\left(\mathbb{R}^{n}\right):\|b\|_{\text {BMO }}<\infty\right\} .
$$

We define the fractional maximal and integral operators.

\section{Definition 3}

(1) Let $0 \leq \alpha<n$,

$$
M_{\alpha} f(x):=\sup _{Q \ni x} l(Q)^{\alpha} f_{Q}|f(y)| d y,
$$

where the supremum is taken over all cubes $Q \subset \mathbb{R}^{n}$ such that $x \in Q$.

(2) Let $0<\alpha<n$,

$$
I_{\alpha} f(x):=\int_{\mathbb{R}^{n}} \frac{f(y)}{|x-y|^{n-\alpha}} d y .
$$


The point-wise inequality holds:

$$
M_{\alpha} f(x) \leq C I_{\alpha} f(x)
$$

for all positive measurable function $f$.

It is well known that the following inequality holds (see [5]). The celebrated result is called the Adams inequality.

Theorem A Let $0<\alpha<n, 1<p \leq p_{0}<\infty$ and $1<q \leq q_{0}<\infty$. Assume that

$$
\frac{1}{q_{0}}=\frac{1}{p_{0}}-\frac{\alpha}{n} \text { and } \quad \frac{q}{q_{0}}=\frac{p}{p_{0}} .
$$

Then we have

$$
\left\|I_{\alpha} f\right\|_{\mathcal{M}_{q}^{q_{0}}} \leq C\|f\|_{\mathcal{M}_{p}^{p_{0}}}
$$

for all $f \in \mathcal{M}_{p}^{p_{0}}\left(\mathbb{R}^{n}\right)$.

Let $m \in \mathbb{Z}_{+}$. The $m$-fold commutator $\left[b, I_{\alpha}\right]^{(m)}$ is given by the following definition.

Definition 4 Let $0<\alpha<n$ and $b \in L_{\text {loc }}^{1}\left(\mathbb{R}^{n}\right)$. Then we define

$$
\left[b, I_{\alpha}\right]^{(m)} f(x):=\int_{\mathbb{R}^{n}} \frac{(b(x)-b(y))^{m}}{|x-y|^{n-\alpha}} f(y) d y,
$$

as long as the integral in the right-hand side makes sense.

Remark 2 The following inequality holds:

$$
\left|\left[b, I_{\alpha}\right]^{(m)} f(x)\right| \leq \int_{\mathbb{R}^{n}} \frac{|b(x)-b(y)|^{m}}{|x-y|^{n-\alpha}}|f(y)| d y .
$$

As shall be verified in the proof of Theorem 1, we virtually consider the operator

$$
x \mapsto \int_{\mathbb{R}^{n}} \frac{|b(x)-b(y)|^{m}}{|x-y|^{n-\alpha}} f(y) d y
$$

and hence we may assume that the integral defining $\left[b, I_{\alpha}\right]^{(m)} f(x)$ converges for a.e. $x \in \mathbb{R}^{n}$.

Di-Fazio and Ragusa [6] obtained the next theorem.

Theorem B Let $0<\alpha<n, 1<p \leq p_{0}<\infty$ and $1<q \leq q_{0}<\infty$. Assume that

$$
\frac{1}{q_{0}}=\frac{1}{p_{0}}-\frac{\alpha}{n} \text { and } \quad \frac{q}{q_{0}}=\frac{p}{p_{0}}
$$

If $b \in \mathrm{BMO}\left(\mathbb{R}^{n}\right)$, then we have

$$
\left\|\left[b, I_{\alpha}\right]^{(1)} f\right\|_{\mathcal{M}_{q}^{q_{0}}} \leq C\|f\|_{\mathcal{M}_{p}^{p_{0}}} .
$$


Conversely if $n-\alpha$ is an even integer and

$$
\left\|\left[b, I_{\alpha}\right]^{(1)} f\right\|_{\mathcal{M}_{q}^{q_{0}}} \leq C\|f\|_{\mathcal{M}_{p}^{p_{0}}}
$$

then $b \in \mathrm{BMO}\left(\mathbb{R}^{n}\right)$.

Komori and Mizuhara [7] removed the restriction ' $n-\alpha$ is an even integer'.

Theorem C Let $0<\alpha<n, 1<p \leq p_{0}<\infty$ and $0<q \leq q_{0}<\infty$. Assume that

$$
\frac{1}{q_{0}}=\frac{1}{p_{0}}-\frac{\alpha}{n} \text { and } \frac{q}{q_{0}}=\frac{p}{p_{0}}
$$

If $b \in \mathrm{BMO}\left(\mathbb{R}^{n}\right)$, then we have

$$
\left\|\left[b, I_{\alpha}\right]^{(1)} f\right\|_{\mathcal{M}_{q}^{q_{0}}} \leq C\|f\|_{\mathcal{M}_{p}^{p_{0}}} .
$$

Conversely if

$$
\left\|\left[b, I_{\alpha}\right]^{(1)} f\right\|_{\mathcal{M}_{q}^{q_{0}}} \leq C\|f\|_{\mathcal{M}_{p}^{p_{0}}}
$$

then $b \in \mathrm{BMO}\left(\mathbb{R}^{n}\right)$.

Sawano et al. [2] proved the following inequality.

Theorem D Let $0<\alpha<n, 1<p \leq p_{0}<\infty, 1<q \leq q_{0}<\infty$ and $1<r \leq r_{0}<\infty$. Assume that

$$
q<r, \quad \frac{1}{p_{0}}>\frac{\alpha}{n} \geq \frac{1}{r_{0}}, \quad \frac{1}{q_{0}}=\frac{1}{p_{0}}+\frac{1}{r_{0}}-\frac{\alpha}{n} \quad \text { and } \quad \frac{q}{q_{0}}=\frac{p}{p_{0}} \text {. }
$$

Suppose that $v \in \mathcal{M}_{r}^{r_{0}}\left(\mathbb{R}^{n}\right)$. Then, for $b \in \mathrm{BMO}\left(\mathbb{R}^{n}\right)$, we have

$$
\left\|\left(\left[b, I_{\alpha}\right]^{(m)} f\right) v\right\|_{\mathcal{M}_{q}^{q_{0}}} \leq C\|b\|_{\mathrm{BMO}}^{m}\|v\|_{\mathcal{M}_{r}^{r_{0}}}\|f\|_{\mathcal{M}_{p}^{p_{0}}} .
$$

In the case of $m=0$, we refer to $[1,8,9]$. In this paper, we generalize Theorem $\mathrm{D}$ to a weighted setting. On the other hand, in [1], the following theorem is proved.

Theorem E Let $0<\alpha<n, 1<p \leq p_{0}<\infty$ and $1<q \leq q_{0}<r_{0}<\infty$. Assume that

$$
\frac{1}{p_{0}}>\frac{\alpha}{n} \geq \frac{1}{r_{0}}, \quad \frac{1}{q_{0}}=\frac{1}{p_{0}}+\frac{1}{r_{0}}-\frac{\alpha}{n}, \quad \frac{q}{q_{0}}=\frac{p}{p_{0}}
$$

and $1<a<\frac{r_{0}}{q_{0}}$. Suppose that the weights $v$ and $w$ satisfy the following condition:

$$
\begin{aligned}
{[v, w]_{a q_{0}, r_{0}, a q, p / a} } & :=\sup _{Q \subset Q^{\prime}}\left(\frac{|Q|}{\left|Q^{\prime}\right|}\right)^{\frac{1}{a q_{0}}}\left|Q^{\prime}\right|^{\frac{1}{r_{0}}}\left(f_{Q} v(x)^{a q} d x\right)^{\frac{1}{a q}}\left(f_{Q^{\prime}} w(x)^{-(p / a)^{\prime}} d x\right)^{\frac{1}{(p / a)^{\prime}}} \\
& <\infty
\end{aligned}
$$


Then we have

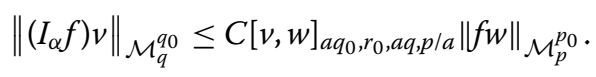

In this paper, we investigate the boundedness of higher order commutators generated by BMO-functions and the fractional integral operator on Morrey spaces corresponding to Theorem E.

\section{Main results and their corollaries}

In this paper, we obtain two main theorems.

\subsection{One of the main results}

Theorem 1 Let $0<\alpha<n, 1<p \leq p_{0}<\infty$ and $1<q \leq q_{0}<r_{0}<\infty$. Assume that

$$
\frac{1}{p_{0}}>\frac{\alpha}{n} \geq \frac{1}{r_{0}}, \quad \frac{1}{q_{0}}=\frac{1}{p_{0}}+\frac{1}{r_{0}}-\frac{\alpha}{n}, \quad \frac{q}{q_{0}}=\frac{p}{p_{0}}
$$

and $1<a<\frac{r_{0}}{q_{0}}$. Suppose that the weights $v$ and $w$ satisfy the condition (2). Then, for $b \in$ $\mathrm{BMO}\left(\mathbb{R}^{n}\right)$, we have

$$
\left\|\left(\left[b, I_{\alpha}\right]^{(m)} f\right) v\right\|_{\mathcal{M}_{q}^{q_{0}}} \leq C\|b\|_{\mathrm{BMO}}^{m}[v, w]_{a q_{0}, r_{0}, a q, p / a}\|f w\|_{\mathcal{M}_{p}^{p_{0}}} .
$$

Remark 3 The condition of Theorem 1 corresponds with the condition of Theorem E. This implies that Theorem 1 gives us the same type of corollaries as in Theorem E.

Taking $w(x)=M_{\frac{a q}{r_{0}} n}\left(v^{a q}\right)(x)^{\frac{1}{a q}}$, we have the following corollary.

Corollary 1 Let $0<\alpha<n, 1<p \leq p_{0}<\infty$ and $1<q \leq q_{0}<r_{0}<\infty$. Assume that

$$
\frac{1}{p_{0}}>\frac{\alpha}{n} \geq \frac{1}{r_{0}}, \quad \frac{1}{q_{0}}=\frac{1}{p_{0}}+\frac{1}{r_{0}}-\frac{\alpha}{n}, \quad \frac{q}{q_{0}}=\frac{p}{p_{0}}
$$

and $1<a<\frac{r_{0}}{q_{0}}$. Let $v$ be a weight. Suppose that $b \in \mathrm{BMO}\left(\mathbb{R}^{n}\right)$, then we have

$$
\left\|\left(\left[b, I_{\alpha}\right]^{(m)} f\right) v\right\|_{\mathcal{M}_{q}^{q_{0}}} \leq C\|b\|_{\mathrm{BMO}}^{m}\left\|f M_{\frac{a q}{r_{0}} n}\left(v^{a q}\right)^{\frac{1}{a q}}\right\|_{\mathcal{M}_{p}^{p_{0}}} .
$$

Taking $w(x) \equiv 1$, we obtain the following corollary.

Corollary 2 Let $0<\alpha<n, 1<p \leq p_{0}<\infty$ and $1<q \leq q_{0}<r_{0}<\infty$. Assume that

$$
\frac{1}{p_{0}}>\frac{\alpha}{n} \geq \frac{1}{r_{0}}, \quad \frac{1}{q_{0}}=\frac{1}{p_{0}}+\frac{1}{r_{0}}-\frac{\alpha}{n}, \quad \frac{q}{q_{0}}=\frac{p}{p_{0}}
$$

and $1<a<\frac{r_{0}}{q_{0}}$. Suppose that $v \in \mathcal{M}_{a q}^{r_{0}}\left(\mathbb{R}^{n}\right)$. Then, for $b \in \mathrm{BMO}\left(\mathbb{R}^{n}\right)$, we have

$$
\left\|\left(\left[b, I_{\alpha}\right]^{(m)} f\right) v\right\|_{\mathcal{M}_{q}^{q_{0}}} \leq C\|b\|_{\mathrm{BMO}}^{m}\|v\|_{\mathcal{M}_{a q}^{r_{a}}}\|f\|_{\mathcal{M}_{p}^{p_{0}}} .
$$

On the other hand, letting $r_{0} \rightarrow \infty$, we obtain the weighted Adams type inequality for the $m$-fold commutator $\left[b, I_{\alpha}\right]^{(m)}$. 
Corollary 3 Let $0<\alpha<n, 1<p \leq p_{0}<\infty$ and $1<q \leq q_{0}<\infty$. Assume that

$$
\frac{1}{q_{0}}=\frac{1}{p_{0}}-\frac{\alpha}{n}, \quad \frac{q}{q_{0}}=\frac{p}{p_{0}}
$$

and $1<a<\frac{r_{0}}{q_{0}}$. Suppose that the weights $v$ and $w$ satisfy the following condition:

$$
[v, w]_{a q_{0}, a q, p / a}:=\sup _{Q \subset Q^{\prime}}\left(\frac{|Q|}{\left|Q^{\prime}\right|}\right)^{\frac{1}{a q_{0}}}\left(f_{Q} v(x)^{a q} d x\right)^{\frac{1}{a q}}\left(f_{Q^{\prime}} w(x)^{-(p / a)^{\prime}} d x\right)^{\frac{1}{(p / a)^{\prime}}}<\infty .
$$

Then, for $b \in \mathrm{BMO}\left(\mathbb{R}^{n}\right)$, we have

$$
\left\|\left(\left[b, I_{\alpha}\right]^{(m)} f\right) v\right\|_{\mathcal{M}_{q}^{q_{0}}} \leq C\|b\|_{\mathrm{BMO}}^{m}[v, w]_{a q_{0}, a q, p / a}\|f w\|_{\mathcal{M}_{p}^{p_{0}}} .
$$

Corollary 3 gives us the following inequality in letting $p=p_{0}, q=q_{0}$ and $v=w$.

Corollary 4 Let $0<\alpha<n, 1<p<\frac{n}{\alpha}$ and $1<q<\infty$. Assume that

$$
\frac{1}{q}=\frac{1}{p}-\frac{\alpha}{n}
$$

Suppose that $w \in A_{p, q}\left(\mathbb{R}^{n}\right)$, i.e.

$$
[w]_{A_{p, q}\left(\mathbb{R}^{n}\right)}:=\sup _{Q \subset \mathbb{R}^{n}}\left(f_{Q} w(x)^{q} d x\right)^{\frac{1}{q}}\left(f_{Q} w(x)^{-p^{\prime}} d x\right)^{\frac{1}{p^{\prime}}}<\infty .
$$

Then, for $b \in \mathrm{BMO}\left(\mathbb{R}^{n}\right)$, we have

$$
\left\|\left(\left[b, I_{\alpha}\right]^{(m)} f\right)\right\|_{L^{q}\left(w^{q}\right)} \leq C\|b\|_{\mathrm{BMO}}^{m}[w]_{A_{p, q}\left(\mathbb{R}^{n}\right)}\|f\|_{L^{p}\left(w^{p}\right)} \quad(m=0,1,2, \ldots) .
$$

Corollary 3 and Theorem $\mathrm{C}$ give us the following corollary.

Corollary 5 Let $0<\alpha<n, 1<p \leq p_{0}<\infty$ and $1<q \leq q_{0}<\infty$. Assume that

$$
\frac{1}{q_{0}}=\frac{1}{p_{0}}-\frac{\alpha}{n}, \quad \frac{q}{q_{0}}=\frac{p}{p_{0}}
$$

and $a>1$. Suppose that the weights $v$ and $w$ satisfy the condition (3). If

$$
\left\|\left[b, I_{\alpha}\right]^{(1)} f\right\|_{\mathcal{M}_{q}^{q_{0}}} \leq C\|f\|_{\mathcal{M}_{p}^{p_{0}}}
$$

holds, then we have for $b \in \mathrm{BMO}\left(\mathbb{R}^{n}\right)$,

$$
\left\|\left(\left[b, I_{\alpha}\right]^{(m)} f\right) v\right\|_{\mathcal{M}_{q}^{q_{0}}} \leq C\|b\|_{\mathrm{BMO}}^{m}[v, w]_{a q_{0}, a q, p / a}\|f w\|_{\mathcal{M}_{p}^{p_{0}}} .
$$

According to Theorem 1.8 in [2], we can pass our result to the operator given by

$$
\left[\vec{b}, I_{\alpha}\right] f(x):=\int_{\mathbb{R}^{n}} \frac{f(y)}{|x-y|^{n-\alpha}} \prod_{j=1}^{m}\left(b_{j}(x)-b_{j}(y)\right) d y,
$$


where $\vec{b}=\left(b_{1}, \ldots, b_{m}\right)$. By a similar argument to [2], as a consequence of Theorem 1 in this paper, we can obtain the following estimate.

Corollary 6 Let $0<\alpha<n, 1<p \leq p_{0}<\infty$ and $1<q \leq q_{0}<r_{0}<\infty$. Assume that

$$
\frac{1}{p_{0}}>\frac{\alpha}{n} \geq \frac{1}{r_{0}}, \quad \frac{1}{q_{0}}=\frac{1}{p_{0}}+\frac{1}{r_{0}}-\frac{\alpha}{n}, \quad \frac{q}{q_{0}}=\frac{p}{p_{0}}
$$

and $1<a<\frac{r_{0}}{q_{0}}$. Suppose that the weights $v$ and $w$ satisfy the condition (2). Then, for $\vec{b}=$ $\left(b_{1}, \ldots, b_{m}\right) \in \mathrm{BMO}\left(\mathbb{R}^{n}\right) \times \cdots \times \mathrm{BMO}\left(\mathbb{R}^{n}\right)$, we have

$$
\left\|\left(\left[\vec{b}, I_{\alpha}\right] f\right) v\right\|_{\mathcal{M}_{q}^{q_{0}}} \leq C\left(\prod_{j=1}^{m}\left\|b_{j}\right\|_{\mathrm{BMO}}\right)[v, w]_{a q_{0}, r_{0}, a q, p / a}\|f w\|_{\mathcal{M}_{p}^{p_{0}}} .
$$

\subsection{Fractional integral operators having rough kernel}

We define the following operators (see [10-12] and [4]).

Definition 5 Let $0<\alpha<n$, a measurable function $\Omega$ on $\mathbb{R}^{n} \backslash\{0\}$ and a measurable function $b$. Then we define

$$
I_{\Omega, \alpha} f(x):=\int_{\mathbb{R}^{n}} \frac{\Omega(x-y) f(y)}{|x-y|^{n-\alpha}} d y
$$

and

$$
\left[b, I_{\Omega, \alpha}\right]^{(m)} f(x):=\int_{\mathbb{R}^{n}} \frac{\Omega(x-y)(b(x)-b(y))^{m} f(y)}{|x-y|^{n-\alpha}} d y .
$$

Remark 4 The following inequality holds:

$$
\left|\left[b, I_{\Omega, \alpha}\right]^{(m)} f(x)\right| \leq \int_{\mathbb{R}^{n}} \frac{|\Omega(x-y)||b(x)-b(y)|^{m}}{|x-y|^{n-\alpha}}|f(y)| d y .
$$

As shall be verified in the proof of Theorem 2, we consider the operator

$$
x \mapsto \int_{\mathbb{R}^{n}} \frac{|\Omega(x-y)||b(x)-b(y)|^{m}}{|x-y|^{n-\alpha}} f(y) d y
$$

and hence we may assume that the integral defining $\left[b, I_{\Omega, \alpha}\right]^{(m)} f(x)$ converges for a.e. $x \in \mathbb{R}^{n}$.

By a similar argument to the proof of Theorem 1, we have the following estimate.

Theorem 2 Let $1<s \leq \infty, 0<\alpha<n, 1 \leq s^{\prime}<p \leq p_{0}<\infty, 1<q \leq q_{0}<\infty$ and $1<r \leq$ $r_{0}<\infty$. Assume that

$$
\frac{1}{p_{0}}>\frac{\alpha}{n} \geq \frac{1}{r_{0}}, \quad \frac{1}{q_{0}}=\frac{1}{p_{0}}+\frac{1}{r_{0}}-\frac{\alpha}{n}, \quad \frac{q}{q_{0}}=\frac{p}{p_{0}}
$$

and $1<a<\frac{r_{0}}{q_{0}}$. Suppose that the weights $v$ and $w$ satisfy $\left[v^{s^{\prime}}, w^{s^{\prime}}\right]_{\frac{a q_{0}}{s^{\prime}}, \frac{r_{0}}{s^{\prime}}, \frac{a q}{s^{\prime}}, \frac{p}{s^{\prime} a}}<\infty$. Moreover, suppose that $\Omega \in L^{s}\left(\mathbb{S}^{n-1}\right)$ is homogeneous of order 0 : For any $\lambda>0, \Omega(\lambda x)=\Omega(x)$. Then, for 
$b \in \operatorname{BMO}\left(\mathbb{R}^{n}\right)$, we have

$$
\left\|\left(\left[b, I_{\Omega, \alpha}\right]^{(m)} f\right) v\right\|_{\mathcal{M}_{q}^{q_{0}}} \leq C\|b\|_{\mathrm{BMO}}^{m}\|\Omega\|_{L^{s}\left(\mathbb{S}^{n-1}\right)}\left[v^{s^{\prime}}, w^{s^{s^{\prime}}}\right]_{\frac{a q_{0}}{s^{\prime}}, \frac{r_{0}}{s^{\prime}}, \frac{a q}{s^{\prime}}, \frac{p}{s^{\prime} a}}\|f w\|_{\mathcal{M}_{p}^{p_{0}}} .
$$

Since $\left[b, I_{\Omega, \alpha}\right]^{(0)}=I_{\Omega, \alpha}$, we refer to [12]. Theorem 2 recovers the following result (see $[4,11])$.

Corollary 7 Let $1<s \leq \infty, 0<\alpha<n, 1 \leq s^{\prime}<p<\frac{n}{\alpha}$ and $1<q<\infty$. Assume that

$$
\frac{1}{q}=\frac{1}{p}-\frac{\alpha}{n}
$$

and $w^{s^{\prime}} \in A_{\frac{p}{s^{\prime}},}, \frac{q}{s^{\prime}}\left(\mathbb{R}^{n}\right)$. Suppose that $\Omega \in L^{s}\left(\mathbb{S}^{n-1}\right)$ is homogeneous of order 0 : For any $\lambda>0$, $\Omega(\lambda x)=\Omega(x)$. Then we have, for $b \in \mathrm{BMO}\left(\mathbb{R}^{n}\right)$,

$$
\left\|\left(\left[b, I_{\Omega, \alpha}\right]^{(m)} f\right)\right\|_{L^{q}\left(w^{q)}\right)} \leq C\left[w^{s^{\prime}}\right]_{\frac{p}{s^{\prime}, \frac{q}{s^{\prime}}}}^{\frac{1}{s^{\prime}}}\left(\mathbb{R}^{n}\right)\|b\|_{\mathrm{BMO}}^{m}\|\Omega\|_{L^{s}\left(\mathbb{S}^{n-1}\right)}\|f\|_{L^{p}\left(w^{p}\right)} .
$$

\section{Some lemmas}

In this section, we prepare some lemmas for proving main results. We recall the following inequalities (see $[3,13]$ and $[4])$.

Lemma 1 (The John-Nirenberg inequality) Let $1 \leq p<\infty$ and let $Q$ be a cube. Then there exists a constant $C>0$ such that

$$
\left(f_{Q}\left|b(x)-m_{Q}(b)\right|^{p} d x\right)^{\frac{1}{p}} \leq C\|b\|_{\text {вмо }}
$$

for all $b \in \mathrm{BMO}\left(\mathbb{R}^{n}\right)$.

We invoke the following decomposition which is derived in [14-16]. We omit the details; see $[1,12]$ for the proof.

Let $\mathcal{D}\left(Q_{0}\right)$ be the collection of all dyadic subcubes of $Q_{0}$, that is, all those cubes obtained by dividing $Q_{0}$ into $2^{n}$ congruent cubes of half its length, dividing each of those into $2^{n}$ congruent cubes. By convention $Q_{0}$ itself to $\mathcal{D}\left(Q_{0}\right)$, and so on.

Lemma 2 Let $\gamma:=m_{3 Q_{0}}(f)$ and $A>2 \cdot 18^{n}$. For $k=1,2, \ldots$ we take

$$
D_{k}:=\bigcup\left\{Q \in \mathcal{D}\left(Q_{0}\right): m_{3 Q}(f)>\gamma A^{k}\right\} .
$$

For $\theta_{1}>1$, let

$$
\gamma^{\prime}:=\left(f_{3 Q_{0}}|f(y)|^{\theta_{1}} d y\right)^{\frac{1}{\theta_{1}}}
$$

and $A^{\prime}>\left(2 \cdot 18^{n}\right)^{\frac{1}{\theta_{1}}}$. For $k=1,2, \ldots$ we take

$$
D_{k}^{\prime}:=\bigcup\left\{Q \in \mathcal{D}\left(Q_{0}\right):\left(f_{3 Q}|f(y)|^{\theta_{1}} d y\right)^{\frac{1}{\theta_{1}}}>\gamma^{\prime} A^{\prime k}\right\} .
$$


Considering the maximality cube, we have

$$
D_{k}=\bigcup_{j} Q_{k, j} \text { and } D_{k}^{\prime}=\bigcup_{j} Q_{k, j}^{\prime}
$$

Then we have

$$
\gamma A^{k}<m_{3 Q_{k, j}}(f) \leq 2^{n} \gamma A^{k} \quad \text { and } \quad \gamma^{\prime} A^{\prime k}<\left(f_{3 Q_{k, j}^{\prime}}|f(y)|^{\theta_{1}} d y\right)^{\frac{1}{\theta_{1}}} \leq 2^{\frac{n}{\theta_{1}}} \gamma^{\prime} A^{k}
$$

Let $E_{k, j}:=Q_{k, j} \backslash D_{k+1}$ and $E_{k, j}^{\prime}:=Q_{k, j}^{\prime} \backslash D_{k+1}^{\prime}$. Moreover we obtain

$$
\left|Q_{k, j}\right| \leq 2\left|E_{k, j}\right| \text { and }\left|Q_{k, j}^{\prime}\right| \leq 2\left|E_{k, j}^{\prime}\right| \cdot
$$

Lemma 3 Under the condition of Theorem 1 , we can choose auxiliary indices $\theta_{1}, \theta_{2}, \theta_{3}, \theta_{4}$. and $\theta_{5}$ so that the following conditions hold:

1. $\theta_{1}, \theta_{2}, \theta_{3}, \theta_{4}$ and $\theta_{5} \in(1, p)$.

2. $L>1$ and $s \in(q, r)$ such that $s \theta_{2}<L q$ and $s^{\prime} \theta_{2}<q^{\prime}$.

3. For the index $\theta_{1} \in(1, p)$, we can choose $a_{*}>1$ such that $a_{*} \theta_{1}<p$.

Assume in addition that, for these indices,

$$
a \geq \max \left\{\theta_{4}, L, \frac{p}{\left(\theta_{5}\left(\frac{p}{\theta_{5}}\right)^{\prime}\right)^{\prime}}, \frac{p}{\left(\theta_{1}\left(\frac{p}{\theta_{1} a_{*}}\right)^{\prime}\right)^{\prime}}, \theta_{3}\right\}>1 \text {. }
$$

Then we obtain

$$
\max \left\{\theta_{5}\left(\frac{p}{\theta_{5}}\right)^{\prime}, \theta_{1}\left(\frac{p}{\theta_{1} a_{*}}\right)^{\prime}\right\} \leq\left(\frac{p}{a}\right)^{\prime}
$$

Proof We examine the second item; $s \theta_{2}<L q$ and $s^{\prime} \theta_{2}<q^{\prime}$. For $0<\varepsilon<1$, we take $\delta=\frac{\varepsilon}{q^{2}}<\varepsilon$. If $s=q+\varepsilon$ and $\theta_{2}=1+\delta$, then we have the following estimate:

$$
\begin{aligned}
s \theta_{2} & =(q+\varepsilon)(1+\delta)=q+q \delta+\varepsilon+\varepsilon \delta \\
& \leq q+q \max \{\varepsilon, \delta\}+\max \{\varepsilon, \delta\}+\max \{\varepsilon, \delta\}^{2} \\
& <q+q \max \{\varepsilon, \delta\}+2 \max \{\varepsilon, \delta\} \\
& <q+q \max \{\varepsilon, \delta\}+2 q \max \{\varepsilon, \delta\} \\
& =q(1+3 \max \{\varepsilon, \delta\})=q(1+3 \varepsilon)=L q .
\end{aligned}
$$

On the other hand, we check $s^{\prime} \theta_{2}<q^{\prime}$ :

$$
q^{\prime}-s^{\prime} \theta_{2}=\frac{\frac{\varepsilon^{2}}{q^{2}}+\frac{\varepsilon}{q}(1-\varepsilon)}{(q-1)(q+\varepsilon-1)}>0
$$

Next we check $\frac{p}{\left(\theta_{5}\left(\frac{p}{\theta_{5}}\right)^{\prime}\right)^{\prime}}>1$. Since $\theta_{5}>1$, we obtain

$$
\theta_{5}\left(\frac{p}{\theta_{5}}\right)^{\prime}>\left(\frac{p}{\theta_{5}}\right)^{\prime}>p^{\prime}
$$


Therefore we have

$$
\left(\theta_{5}\left(\frac{p}{\theta_{5}}\right)^{\prime}\right)^{\prime}<\left(p^{\prime}\right)^{\prime}=p .
$$

This gives us

$$
\frac{p}{\left(\theta_{5}\left(\frac{p}{\theta_{5}}\right)^{\prime}\right)^{\prime}}>1
$$

By a similar argument, we obtain

$$
\frac{p}{\left(\theta_{1}\left(\frac{p}{\theta_{1} a_{*}}\right)^{\prime}\right)^{\prime}}>1 \text {. }
$$

Remark 5 The index $\theta_{1}$ in Lemma 2 corresponds with the index $\theta_{1}$ in Lemma 3.

\section{Proof of Theorem 1}

Proof of Theorem 1 Fix a dyadic cube $Q_{0} \in \mathcal{D}\left(\mathbb{R}^{n}\right)$. Let $\mathcal{D}_{\nu}$ be the collection of dyadic cubes.

The volume of the elements of $\mathcal{D}_{v}$ is $2^{n v}$. For $x \in Q_{0}$, we have

$$
\begin{aligned}
\left|\left[b, I_{\alpha}\right]^{(m)} f(x)\right| & \leq C \sum_{\nu \in \mathbb{Z}} \sum_{\substack{Q \in \mathcal{D}_{\nu},|Q|=2^{v n}}} 2^{-v(n-\alpha)} \chi_{Q}(x) \int_{3 Q}|b(x)-b(y)|^{m}|f(y)| d y \\
& =C \sum_{\nu \in \mathbb{Z}}\left(\sum_{\substack{Q \in \mathcal{D}_{\nu}, Q \subseteq Q_{0}}}+\sum_{\substack{Q \in \mathcal{D}_{v}, Q \supsetneq Q_{0}}}\right) 2^{-v(n-\alpha)} \chi_{Q}(x) \int_{3 Q}|b(x)-b(y)|^{m}|f(y)| d y \\
& =: C(A+B) .
\end{aligned}
$$

We evaluate $A$ and $B$ in Sections 4.1 and 4.2, respectively.

\subsection{The estimate of $\boldsymbol{A}$}

By $|b(x)-b(y)|^{m} \leq 2^{m-1}\left(\left|b(x)-m_{Q}(b)\right|^{m}+\left|m_{Q}(b)-b(y)\right|^{m}\right)$, we obtain

$$
\begin{aligned}
A= & \sum_{Q \in \mathcal{D}\left(Q_{0}\right)} l(Q)^{\alpha-n} \chi_{Q}(x) \int_{3 Q}|b(x)-b(y)|^{m}|f(y)| d y \\
\leq & C \sum_{Q \in \mathcal{D}\left(Q_{0}\right)} l(Q)^{\alpha} \chi_{Q}(x)\left|b(x)-m_{Q}(b)\right|^{m} f_{3 Q}|f(y)| d y \\
& +C \sum_{Q \in \mathcal{D}\left(Q_{0}\right)} l(Q)^{\alpha} \chi_{Q}(x) f_{3 Q}\left|m_{Q}(b)-b(y)\right|^{m}|f(y)| d y .
\end{aligned}
$$

We take $\theta_{1}>1$ as in Lemma 2. By Hölder's inequality for $\theta_{1}>1$, we have

$$
\begin{aligned}
A \leq & C \sum_{Q \in \mathcal{D}\left(Q_{0}\right)} l(Q)^{\alpha} \chi_{Q}(x)\left|b(x)-m_{Q}(b)\right|^{m} f_{3 Q}|f(y)| d y \\
& +C \sum_{Q \in \mathcal{D}\left(Q_{0}\right)} l(Q)^{\alpha} \chi_{Q}(x)\left(f_{3 Q}\left|m_{Q}(b)-b(y)\right|^{m \theta_{1}^{\prime}} d y\right)^{\frac{1}{\theta_{1}^{\prime}}}\left(f_{3 Q}|f(y)|^{\theta_{1}} d y\right)^{\frac{1}{\theta_{1}}} .
\end{aligned}
$$


By Lemma 1, we have

$$
\begin{aligned}
A \leq & C \sum_{Q \in \mathcal{D}\left(Q_{0}\right)} l(Q)^{\alpha} \chi_{Q}(x)\left|b(x)-m_{Q}(b)\right|^{m} f_{3 Q}|f(y)| d y \\
& +C\|b\|_{\mathrm{BMO}}^{m} \sum_{Q \in \mathcal{D}\left(Q_{0}\right)} l(Q)^{\alpha} \chi_{Q}(x)\left(f_{3 Q}|f(y)|^{\theta_{1}} d y\right)^{\frac{1}{\theta_{1}}} \\
= & C\left(I+\|b\|_{\mathrm{BMO}}^{m} I I\right) .
\end{aligned}
$$

We evaluate $I$. Let

$$
\mathcal{D}_{0}\left(Q_{0}\right):=\left\{Q \in \mathcal{D}\left(Q_{0}\right) ;\left(f_{3 Q}|f(y)| d y\right) \leq \gamma A\right\}
$$

and

$$
\mathcal{D}_{k, j}\left(Q_{0}\right):=\left\{Q \in \mathcal{D}\left(Q_{0}\right) ; Q \subset Q_{k, j}, \gamma A^{k}<\left(f_{3 Q}|f(y)| d y\right) \leq \gamma A^{k+1}\right\},
$$

where $Q_{k, j}$ is in Lemma 2. Then we have

$$
\mathcal{D}\left(Q_{0}\right)=\mathcal{D}_{0}\left(Q_{0}\right) \cup\left(\bigcup_{k, j} \mathcal{D}_{k, j}\left(Q_{0}\right)\right)
$$

By the duality argument, we have

$$
\left(\int_{Q_{0}} I^{q} \cdot v(x)^{q} d x\right)^{\frac{1}{q}}=\sup _{\|g\|_{L^{\prime}}\left(Q_{0}\right)}\left(\int_{Q_{0}} I \cdot v(x)|g(x)| d x\right) .
$$

Let $g \geq 0, \operatorname{supp}(g) \subset Q_{0},\|g\|_{L^{q^{\prime}}\left(Q_{0}\right)}=1$. Then we have

$$
\begin{aligned}
\int_{Q_{0}} I \cdot v(x)|g(x)| d x \leq & C \sum_{Q \in \mathcal{D}\left(Q_{0}\right)} l(Q)^{\alpha}\left(f_{3 Q}|f(y)| d y\right) \\
& \times \int_{Q}\left|b(x)-m_{Q}(b)\right|^{m} v(x) g(x) d x \\
= & C\left(\sum_{Q \in \mathcal{D}_{0}\left(Q_{0}\right)}+\sum_{k, j} \sum_{Q \in \mathcal{D}_{k, j}\left(Q_{0}\right)}\right) l(Q)^{\alpha}\left(f_{3 Q}|f(y)| d y\right) \\
& \times \int_{Q}\left|b(x)-m_{Q}(b)\right|^{m} v(x) g(x) d x \\
= & I_{0}+\sum_{k, j} I_{k, j} .
\end{aligned}
$$

We evaluate $I_{k, j}$. If $Q \in \mathcal{D}_{k, j}\left(Q_{0}\right)$, then we have

$$
f_{3 Q}|f(y)| d y \leq \gamma A^{k+1}
$$


Hence we obtain

$$
\begin{aligned}
I_{k, j} & \leq \sum_{Q \in \mathcal{D}_{k, j}\left(Q_{0}\right)} l(Q)^{\alpha} \gamma A^{k+1} \int_{Q}\left|b(x)-m_{Q}(b)\right|^{m} v(x) g(x) d x \\
& \leq A \sum_{Q \in \mathcal{D}_{k, j}\left(Q_{0}\right)} l\left(Q_{k, j}\right)^{\alpha} \gamma A^{k} \int_{Q}\left|b(x)-m_{Q}(b)\right|^{m} v(x) g(x) d x .
\end{aligned}
$$

Since

$$
\gamma A^{k}<f_{3 Q_{k, j}}|f(y)| d y
$$

we obtain

$$
I_{k, j} \leq A \sum_{Q \in \mathcal{D}_{k, j}\left(Q_{0}\right)} l\left(Q_{k, j}\right)^{\alpha} f_{3 Q_{k, j}}|f(y)| d y \int_{Q}\left|b(x)-m_{Q}(b)\right|^{m} v(x) g(x) d x .
$$

By Hölder's inequality for $\theta_{2}>1$ as in Lemma 3, we obtain

$$
\begin{aligned}
I_{k, j} \leq & A l\left(Q_{k, j}\right)^{\alpha} m_{3 Q_{k, j}}(f \mid) \sum_{Q \in \mathcal{D}_{k, j}\left(Q_{0}\right)}\left(\int_{Q}\left|b(x)-m_{Q}(b)\right|^{m} v(x) g(x) d x\right) \\
\leq & A l\left(Q_{k, j}\right)^{\alpha} m_{3 Q_{k, j}}(|f|) \sum_{Q \in \mathcal{D}_{k, j}\left(Q_{0}\right)}|Q|\left(f_{Q}\left|b(x)-m_{Q}(b)\right|^{m \theta_{2}^{\prime}} d x\right)^{\frac{1}{\theta_{2}^{\prime}}} \\
& \times\left(f_{Q} v(x)^{\theta_{2}} g(x)^{\theta_{2}} d x\right)^{\frac{1}{\theta_{2}}} .
\end{aligned}
$$

By Lemma 1, we obtain

$$
\begin{aligned}
I_{k, j} & \leq A\|b\|_{\mathrm{BMO}}^{m} l\left(Q_{k, j}\right)^{\alpha} m_{3 Q_{k, j}}(|f|) \sum_{Q \in \mathcal{D}_{k, j}\left(Q_{0}\right)} \int_{Q}\left(f_{Q}(v(y) g(y))^{\theta_{2}} d y\right)^{\frac{1}{\theta_{2}}} d x \\
& \leq A\|b\|_{\mathrm{BMO}}^{m} l\left(Q_{k, j}\right)^{\alpha} m_{3 Q_{k, j}}(|f|) \sum_{Q \in \mathcal{D}_{k, j}\left(Q_{0}\right)} \int_{Q} M\left[\left(v_{k, j} g\right)^{\theta_{2}}\right](x)^{\frac{1}{\theta_{2}}} d x,
\end{aligned}
$$

where $v_{k, j}=v \chi_{Q_{k, j}}$ and the symbol $M$ is the ordinary Hardy-Littlewood maximal operator. By Lemma 2, we have

$$
\begin{aligned}
I_{k, j} & \leq A\|b\|_{\mathrm{BMO}}^{m}\left|Q_{k, j}\right| l\left(Q_{k, j}\right)^{\alpha} m_{3 Q_{k, j}}(|f|)\left(f_{Q_{k, j}} M\left[\left(v_{k, j} g\right)^{\theta_{2}}\right](x)^{\frac{1}{\theta_{2}}} d x\right) \\
& \leq 2 A\|b\|_{\mathrm{BMO}}^{m}\left|E_{k, j}\right| l\left(Q_{k, j}\right)^{\alpha} m_{3 Q_{k, j}}(|f|)\left(f_{Q_{k, j}} M\left[\left(v_{k, j} g\right)^{\theta_{2}}\right](x)^{\frac{1}{\theta_{2}}} d x\right) \\
& =2 A\|b\|_{\mathrm{BMO}}^{m} \int_{E_{k, j}} l\left(Q_{k, j}\right)^{\alpha} m_{3 Q_{k, j}}(|f|)\left(f_{Q_{k, j}} M\left[\left(v_{k, j} g\right)^{\theta_{2}}\right](x)^{\frac{1}{\theta_{2}}} d x\right) d y .
\end{aligned}
$$

We take $s \in(q, r)$ and $L>1$ as in Lemma 3. By Hölder's inequality for $s>1$, we have

$$
M\left[\left(v_{k, j} g\right)^{\theta_{2}}\right](x)^{\frac{1}{\theta_{2}}} \leq M\left[v_{k, j}^{s \theta_{2}}\right](x)^{\frac{1}{s \theta_{2}}} M\left[g^{s^{\prime} \theta_{2}}\right](x)^{\frac{1}{s^{\prime} \theta_{2}}} .
$$


lido Journal of Inequalities and Applications (2016) 2016:4

Page 13 of 23

By Hölder's inequality for $L q>1$, we obtain the following inequality:

$$
\begin{aligned}
& \left(f_{Q_{k, j}} M\left[\left(v_{k, j} g\right)^{\theta_{2}}\right](x)^{\frac{1}{\theta_{2}}} d x\right) \\
& \quad \leq\left(f_{Q_{k, j}} M\left[v_{k, j}^{s \theta_{2}}\right](x)^{\frac{L q}{s \theta_{2}}} d x\right)^{\frac{1}{L q}}\left(f_{Q_{k, j}} M\left[g^{s^{\prime} \theta_{2}}\right](x)^{\frac{(L q)^{\prime}}{s^{\prime} \theta_{2}}} d x\right)^{\frac{1}{(L q)^{\prime}}} .
\end{aligned}
$$

Since $s \theta_{2}<L q$, the boundedness of $M: L^{\frac{L q}{s \theta_{2}}}\left(\mathbb{R}^{n}\right) \rightarrow L^{\frac{L q}{s \theta_{2}}}\left(\mathbb{R}^{n}\right)$ gives us the following inequality:

$$
\begin{aligned}
& \left(f_{Q_{k, j}} M\left[\left(v_{k, j} g\right)^{\theta_{2}}\right](x)^{\frac{1}{\theta_{2}}} d x\right) \\
& \quad \leq C\left(\frac{1}{\left|Q_{k, j}\right|} \int_{\mathbb{R}^{n}} v_{k, j}(x)^{L q} d x\right)^{\frac{1}{L q}}\left(f_{Q_{k, j}} M\left[g^{s^{\prime} \theta_{2}}\right](x)^{\frac{(L q)^{\prime}}{s^{\prime} \theta_{2}}} d x\right)^{\frac{1}{(L q)^{\prime}}} .
\end{aligned}
$$

Since $a \geq L>1$, by Hölder's inequality for $\frac{a}{L} \geq 1$,

$$
\begin{aligned}
& \left(f_{Q_{k, j}} M\left[\left(v_{k, j} g\right)^{\theta_{2}}\right](x)^{\frac{1}{\theta_{2}}} d x\right) \\
& \quad \leq C\left(f_{Q_{k, j}} v(x)^{a q} d x\right)^{\frac{1}{a q}}\left(f_{Q_{k, j}} M\left[g^{s^{\prime} \theta_{2}}\right](x)^{\frac{(L q)^{\prime}}{s^{\prime} \theta_{2}}} d x\right)^{\frac{1}{(L q)^{\prime}}}
\end{aligned}
$$

By Lemma 2, this implies that

$$
I_{k, j} \leq 2 A\|b\|_{\mathrm{BMO}}^{m} \int_{E_{k, j}} M_{\alpha, a q}(f, v)(x) \cdot M\left[M\left[g^{s^{\prime} \theta_{2}}\right]^{\frac{(L q)^{\prime}}{s^{\prime} \theta_{2}}}\right](x)^{\frac{1}{(L q)^{\prime}}} d x,
$$

where

$$
M_{\alpha, a q}(f, v)(x):=\sup _{Q \ni x} l(Q)^{\alpha} m_{3 Q}(f)\left(f_{Q} v(x)^{a q} d x\right)^{\frac{1}{a q}} .
$$

A similar argument gives us the following estimate:

$$
I_{0} \leq 2 A\|b\|_{\mathrm{BMO}}^{m} \int_{E_{0}} M_{\alpha, a q}(f, v)(x) \cdot M\left[M\left[g^{s^{\prime} \theta_{2}}\right]^{\frac{(L q)^{\prime}}{s^{\prime} \theta_{2}}}\right](x)^{\frac{1}{(L q)^{\prime}}} d x .
$$

By summing up $I_{0}$ and $I_{k, j}$, we obtain

$$
I_{0}+\sum_{k, j} I_{k, j} \leq 2 A\|b\|_{\mathrm{BMO}}^{m} \int_{Q_{0}} M_{\alpha, a q}(f, v)(x) \cdot M\left[M\left[g^{s^{\prime} \theta_{2}}\right]^{\frac{(L q)^{\prime}}{s^{\prime} \theta_{2}}}\right](x)^{\frac{1}{(L q)^{\prime}}} d x
$$

By Hölder's inequality for $q>1$, we have

$$
\begin{aligned}
& \int_{Q_{0}} M_{\alpha, a q}(f, v)(x) \cdot M\left[M\left[g^{s^{\prime} \theta_{2}}\right]^{\frac{(L q)^{\prime}}{s^{\prime} \theta_{2}}}\right](x)^{\frac{1}{(L q)^{\prime}}} d x \\
& \quad \leq\left(\int_{Q_{0}} M_{\alpha, a q}(f, v)(x)^{q} d x\right)^{\frac{1}{q}}\left(\int_{Q_{0}} M\left[M\left[g^{s^{\prime} \theta_{2}}\right]^{\frac{(L q)^{\prime}}{s^{\prime} \theta_{2}}}\right](x)^{\frac{q^{\prime}}{(L q)^{\prime}}} d x\right)^{\frac{1}{q^{\prime}}} .
\end{aligned}
$$


lido Journal of Inequalities and Applications (2016) 2016:4

Page 14 of 23

Since $(L q)^{\prime}<q^{\prime}$, the boundedness of $M: L^{\frac{q^{\prime}}{(L q)^{\prime}}}\left(\mathbb{R}^{n}\right) \rightarrow L^{\frac{q^{\prime}}{(L q)^{\prime}}}\left(\mathbb{R}^{n}\right)$ gives us the following inequality:

$$
\begin{aligned}
\left(\int_{Q_{0}} M\left[M\left[g^{s^{\prime} \theta_{2}}\right]^{\frac{(L q)^{\prime}}{s^{\prime} \theta_{2}}}\right](x)^{\frac{q^{\prime}}{(L q)^{\prime}}} d x\right)^{\frac{1}{q^{\prime}}} & \leq C\left(\int_{\mathbb{R}^{n}} M\left[g^{s^{\prime} \theta_{2}}\right](x)^{\frac{(L q)^{\prime}}{s^{\prime} \theta_{2}} \cdot \frac{q^{\prime}}{(L q)^{\prime}}} d x\right)^{\frac{1}{q^{\prime}}} \\
& =C\left(\int_{\mathbb{R}^{n}} M\left[g^{s^{\prime} \theta_{2}}\right](x)^{\frac{q^{\prime}}{s^{\prime} \theta_{2}}} d x\right)^{\frac{1}{q^{\prime}}}
\end{aligned}
$$

Since $s^{\prime} \theta_{2}<q^{\prime}$, the boundedness of $M: L^{\frac{q^{\prime}}{s^{\prime} \theta_{2}}}\left(\mathbb{R}^{n}\right) \rightarrow L^{\frac{q^{\prime}}{s^{\prime} \theta_{2}}}\left(\mathbb{R}^{n}\right)$ gives us the following inequality:

$$
\begin{aligned}
\left(\int_{\mathbb{R}^{n}} M\left[g^{s^{\prime} \theta_{2}}\right](x)^{\frac{q^{\prime}}{s^{\prime} \theta_{2}}} d x\right)^{\frac{1}{q^{\prime}}} & \leq C\left(\int_{Q_{0}}|g(x)|^{s^{\prime} \theta_{2} \cdot \frac{q^{\prime}}{s^{\prime} \theta_{2}}} d x\right)^{\frac{1}{q^{\prime}}} \\
& =C\left(\int_{Q_{0}}|g(x)|^{q^{\prime}} d x\right)^{\frac{1}{q^{\prime}}}=C .
\end{aligned}
$$

By Hölder's inequality for $\frac{p}{a}>1$, we obtain

$$
M_{\alpha, a q}(f, v)(x) \leq \sup _{Q \ni x} l(Q)^{\alpha} m_{3 Q}\left(|f w|^{\frac{p}{a}}\right)^{\frac{a}{p}}\left(f_{Q} v(y)^{a q} d y\right)^{\frac{1}{a q}}\left(f_{3 Q} w(y)^{-(p / a)^{\prime}} d y\right)^{\frac{1}{(p / a)^{\prime}}}
$$

By the condition (2), we obtain

$$
\begin{aligned}
M_{\alpha, a q}(f, v)(z) & \leq C[v, w]_{a q_{0}, r_{0}, a q, p / a} \sup _{Q \ni z} l(Q)^{\alpha-\frac{n}{r_{0}}} m_{3 Q}\left(|f w|^{\frac{p}{a}}\right)^{\frac{a}{p}} \\
& \left.\leq C[v, w]_{a q_{0}, r_{0}, a q, p / a} M_{\left(\alpha-\frac{n}{r_{0}}\right) \frac{p}{a}}(f w)^{\frac{p}{a}}\right)(z)^{\frac{a}{p}}
\end{aligned}
$$

This implies that

$$
\left|Q_{0}\right|^{\frac{1}{q_{0}}}\left(f_{Q_{0}} M_{\alpha, a q}(f, v)(z)^{q} d z\right)^{\frac{1}{q}} \leq C[v, w]_{a q_{0}, r_{0}, a q, p / a}\left\|M_{\left(\alpha-\frac{n}{r_{0}}\right) \frac{p}{a}}\left((f w)^{\frac{p}{a}}\right)\right\|_{\mathcal{M}_{\frac{\frac{a q}{p}}{p}}^{\frac{a q_{0}}{p}}}
$$

Since

$$
\frac{1}{q_{0}} \cdot \frac{p}{a}=\frac{1}{p_{0}} \cdot \frac{p}{a}-\frac{\left(\alpha-\frac{n}{r_{0}}\right) \cdot \frac{p}{a}}{n} \text { and } \quad \frac{\frac{a p_{0}}{p}}{\frac{a q_{0}}{p}}=\frac{a}{\frac{a q}{p}}
$$

by Theorem A, we have

$$
\begin{aligned}
& \left|Q_{0}\right|^{\frac{1}{q_{0}}}\left(f_{Q_{0}} M_{\alpha, a q}(f, v)(z)^{q} d z\right)^{\frac{1}{q}} \\
& \quad \leq C[v, w]_{a q_{0}, r_{0}, a q, p / a}\left\|(f w)^{\frac{p}{a}}\right\|_{\mathcal{M}_{a}^{\frac{a}{p}}}^{\frac{a p_{0}}{p}} \\
& \quad=C[v, w]_{a q_{0}, r_{0}, a q, p / a}\left(\sup _{Q}|Q|^{\frac{p}{a p_{0}}}\left(f_{Q}|f(x) w(x)|^{\frac{p}{a} \cdot a} d x\right)^{\frac{1}{a}}\right)^{\frac{a}{p}}
\end{aligned}
$$


lido Journal of Inequalities and Applications <wide>(2016) 2016:4

Page 15 of 23

$$
\begin{aligned}
& =C[v, w]_{a q_{0}, r_{0}, a q, p / a} \sup _{Q}|Q|^{\frac{1}{p_{0}}}\left(f_{Q}|f(x)|^{p} w(x)^{p} d x\right)^{\frac{1}{p}} \\
& =C[v, w]_{a q_{0}, r_{0}, a q, p / a}\|f w\|_{\mathcal{M}_{p}^{p_{0}}} .
\end{aligned}
$$

We evaluate $I I$. Let

$$
\mathcal{D}_{0}^{\prime}\left(Q_{0}\right):=\left\{Q \in \mathcal{D}\left(Q_{0}\right) ;\left(f_{3 Q}|f(y)|^{\theta_{1}} d y\right)^{\frac{1}{\theta_{1}}} \leq \gamma^{\prime} A^{\prime}\right\}
$$

and

$$
\mathcal{D}_{k, j}^{\prime}\left(Q_{0}\right):=\left\{Q \in \mathcal{D}\left(Q_{0}\right) ; Q \subset Q_{k, j}^{\prime}, \gamma^{\prime} A^{\prime k}<\left(f_{3 Q}|f(y)|^{\theta_{1}} d y\right)^{\frac{1}{\theta_{1}}} \leq \gamma^{\prime} A^{\prime k+1}\right\}
$$

where $Q_{k, j}^{\prime}$ is found in Lemma 2. Then we have

$$
\mathcal{D}\left(Q_{0}\right)=\mathcal{D}_{0}^{\prime}\left(Q_{0}\right) \cup\left(\bigcup_{k, j} \mathcal{D}_{k, j}^{\prime}\left(Q_{0}\right)\right)
$$

By the duality argument, we have

$$
\left(\int_{Q_{0}} I I^{q} \cdot v(x)^{q} d x\right)^{\frac{1}{q}}=\sup _{\|g\|_{L^{q^{\prime}}\left(Q_{0}\right)}}\left(\int_{Q_{0}} I I \cdot v(x)|g(x)| d x\right) .
$$

Let $g \geq 0$ be such that $\operatorname{supp}(g) \subset Q_{0}$ and $\|g\|_{L^{q^{\prime}}\left(Q_{0}\right)}=1$. We have

$$
\begin{aligned}
\int_{Q_{0}} I I \cdot v(x) g(x) d x & \leq \sum_{Q \in \mathcal{D}\left(Q_{0}\right)} l(Q)^{\alpha}\left(f_{3 Q}|f(y)|^{\theta_{1}} d y\right)^{\frac{1}{\theta_{1}}}\|v g\|_{L^{1}(Q)} \\
& \leq\left(\sum_{Q \in \mathcal{D}_{0}^{\prime}\left(Q_{0}\right)}+\sum_{k, j} \sum_{Q \in \mathcal{D}_{k, j}^{\prime}\left(Q_{0}\right)}\right) l(Q)^{\alpha}\left(f_{3 Q}|f(y)|^{\theta_{1}} d y\right)^{\frac{1}{\theta_{1}}}\|v g\|_{L^{1}(Q)} \\
& \leq\left(I I_{0}+\sum_{k, j} I I_{k, j}\right) .
\end{aligned}
$$

We evaluate $I I_{k, j}$. If $Q \in \mathcal{D}_{k, j}^{\prime}\left(Q_{0}\right)$, then we have

$$
\left(f_{3 Q}|f(y)|^{\theta_{1}} d y\right)^{\frac{1}{\theta_{1}}} \leq \gamma^{\prime} A^{\prime k+1}
$$

Therefore we obtain

$$
\begin{aligned}
I I_{k, j} & \leq \sum_{Q \in \mathcal{D}_{k, j}^{\prime}\left(Q_{0}\right)} l(Q)^{\alpha}\left(f_{3 Q}|f(y)|^{\theta_{1}} d y\right)^{\frac{1}{\theta_{1}}} \int_{Q} v(x) g(x) d x \\
& \leq \gamma^{\prime} A^{\prime k+1} \sum_{Q \in \mathcal{D}_{k, j}^{\prime}\left(Q_{0}\right)} l(Q)^{\alpha} \int_{Q} v(x) g(x) d x .
\end{aligned}
$$


lido Journal of Inequalities and Applications <wide>(2016) 2016:4

Page 16 of 23

Since

$$
\gamma^{\prime} A^{\prime k} \leq\left(f_{3 Q_{k, j}^{\prime}}|f(y)|^{\theta_{1}} d y\right)^{\frac{1}{\theta_{1}}}
$$

we obtain

$$
I I_{k, j} \leq A^{\prime}\left(f_{3 Q_{k, j}^{\prime}}|f(y)|^{\theta_{1}} d y\right)^{\frac{1}{\theta_{1}}} l\left(Q_{k, j}^{\prime}\right)^{\alpha}\left(f_{Q_{k, j}^{\prime}} v(x) g(x) d x\right)\left|Q_{k, j}^{\prime}\right| .
$$

By Hölder's inequality for $\theta_{3}>1$ as in Lemma 3 , we have

$$
\begin{aligned}
I I_{k, j} \leq & A^{\prime}\left(f_{3 Q_{k, j}^{\prime}}|f(y)|^{\theta_{1}} d y\right)^{\frac{1}{\theta_{1}}} l\left(Q_{k, j}^{\prime}\right)^{\alpha}\left(f_{Q_{k, j}^{\prime}} v(x)^{\theta_{3} q} d x\right)^{\frac{1}{\theta_{3} q}} \\
& \times\left(f_{Q_{k, j}^{\prime}} g(x)^{\left(\theta_{3} q\right)^{\prime}} d x\right)^{\frac{1}{\left(\theta_{3} q\right)^{\prime}}}\left|Q_{k, j}^{\prime}\right| .
\end{aligned}
$$

By Lemma 2, we obtain

$$
\begin{aligned}
I I_{k, j} \leq & 2 A^{\prime}\left(f_{3 Q_{k, j}^{\prime}}|f(y)|^{\theta_{1}} d y\right)^{\frac{1}{\theta_{1}}} l\left(Q_{k, j}^{\prime}\right)^{\alpha}\left(f_{Q_{k, j}^{\prime}} v(x)^{\theta_{3} q} d x\right)^{\frac{1}{\theta_{3} q}} \\
& \times\left(f_{Q_{k, j}^{\prime}} g(x)^{\left(\theta_{3} q\right)^{\prime}} d x\right)^{\frac{1}{\left(\theta_{3} q\right)^{\prime}}}\left|E_{k, j}^{\prime}\right| \\
= & 2 A^{\prime} \int_{E_{k, j}^{\prime}} l\left(Q_{k, j}^{\prime}\right)^{\alpha}\left(f_{3 Q_{k, j}^{\prime}}|f(x)|^{\theta_{1}} d x\right)^{\frac{1}{\theta_{1}}}\left(f_{Q_{k, j}^{\prime}} v(x)^{\theta_{3} q} d x\right)^{\frac{1}{\theta_{3} q}} \\
& \times\left(f_{Q_{k, j}^{\prime}} g(x)^{\left(\theta_{3} q\right)^{\prime}} d x\right)^{\frac{1}{\left(\theta_{3} q\right)^{\prime}}} d y \\
\leq & 2 A^{\prime} \int_{E_{k, j}^{\prime}} \tilde{M}_{\alpha, \theta_{1}, \theta_{3} q}\left(f^{\theta_{1}}, v\right)(y) \cdot M\left[g^{\left(\theta_{3} q\right)^{\prime}}\right](y)^{\frac{1}{\left(\theta_{3} q\right)^{\prime}}} d y,
\end{aligned}
$$

where

$$
\tilde{M}_{\alpha, \theta_{1}, \theta_{3} q}\left(f^{\theta_{1}}, v\right)(y):=\sup _{Q \ni y} l(Q)^{\alpha}\left(f_{3 Q}|f(x)|^{\theta_{1}} d x\right)^{\frac{1}{\theta_{1}}}\left(f_{Q} v(x)^{\theta_{3} q} d x\right)^{\frac{1}{\theta_{3} q}} .
$$

A similar argument gives us the following estimate:

$$
I I_{0} \leq 2 A^{\prime} \int_{E_{0}^{\prime}} \tilde{M}_{\alpha, \theta_{1}, \theta_{3} q}\left(f^{\theta_{1}}, v\right)(y) \cdot M\left[g^{\left(\theta_{3} q\right)^{\prime}}\right](y)^{\frac{1}{\left(\theta_{3} q\right)^{\prime}}} d y .
$$

By summing up $I I_{0}$ and $I I_{k, j}$, we obtain

$$
I I_{0}+\sum_{k, j} I I_{k, j} \leq 2 A^{\prime} \int_{Q_{0}} \tilde{M}_{\alpha, \theta_{1}, \theta_{3} q}\left(f^{\theta_{1}}, v\right)(y) \cdot M\left[g^{\left(\theta_{3} q\right)^{\prime}}\right](y)^{\frac{1}{\left(\theta_{3} q\right)^{\prime}}} d y .
$$


By Hölder's inequality for $q>1$, we have

$$
\begin{aligned}
& \int_{\mathrm{Q}_{0}} \tilde{M}_{\alpha, \theta_{1}, \theta_{3} q}\left(f^{\theta_{1}}, v\right)(y) \cdot M\left[g^{\left(\theta_{3} q\right)^{\prime}}\right](y)^{\frac{1}{\left(\theta_{3} q\right)^{\prime}}} d y \\
& \quad \leq\left(\int_{Q_{0}} \tilde{M}_{\alpha, \theta_{1}, \theta_{3} q}\left(f^{\theta_{1}}, v\right)(y)^{q} d y\right)^{\frac{1}{q}} \cdot\left(\int_{Q_{0}} M\left[g^{\left(\theta_{3} q\right)^{\prime}}\right](y)^{\frac{q^{\prime}}{\left(\theta_{3} q\right)^{\prime}}} d y\right)^{\frac{1}{q^{\prime}}} .
\end{aligned}
$$

Since $\left(\theta_{3} q\right)^{\prime}<q^{\prime}$ and $\operatorname{supp}(g) \subset Q_{0}$, by the boundedness of $M: L^{\frac{q^{\prime}}{\left(\theta_{3} q\right)^{\prime}}}\left(\mathbb{R}^{n}\right) \rightarrow L^{\frac{q^{\prime}}{\left(\theta_{3} q\right)^{\prime}}}\left(\mathbb{R}^{n}\right)$, we have

$$
\left(\int_{Q_{0}} M\left[g^{\left(\theta_{3} q\right)^{\prime}}\right](y)^{\frac{q^{\prime}}{\left(\theta_{3} q\right)^{\prime}}} d y\right)^{\frac{1}{q^{\prime}}} \leq C\left(\int_{Q_{0}} g(x)^{\left(\theta_{3} q\right)^{\prime} \cdot \frac{q^{\prime}}{\left(\theta_{3} q\right)^{\prime}}} d x\right)^{\frac{1}{q^{\prime}}}=C .
$$

Therefore we have

$$
\int_{\mathrm{Q}_{0}} \tilde{M}_{\alpha, \theta_{1}, \theta_{3} q}\left(f^{\theta_{1}}, v\right)(y) \cdot M\left[g^{\left(\theta_{3} q\right)^{\prime}}\right](y)^{\frac{1}{\left(\theta_{3} q\right)^{\prime}}} d y \leq C\left(\int_{\mathrm{Q}_{0}} \tilde{M}_{\alpha, \theta_{1}, \theta_{3} q}\left(f^{\theta_{1}}, v\right)(y)^{q} d y\right)^{\frac{1}{q}} .
$$

By Hölder's inequality for $\frac{p}{a_{*} \theta_{1}}>1$ as in Lemma 3, we have

$$
\begin{aligned}
\tilde{M}_{\alpha, \theta_{1}, \theta_{3} q}\left(f^{\theta_{1}}, v\right)(x) \leq & C \sup _{Q \ni x} l(Q)^{\alpha} m_{3 Q}\left(|f w|^{\frac{p}{a_{*}}}\right)^{\frac{a_{*}}{p}} \\
& \times\left(f_{3 Q} w(y)^{-\theta_{1}\left(\frac{p}{\theta_{1} a_{*}}\right)^{\prime}} d y\right)^{\frac{1}{\theta_{1}} \frac{1}{\left(\frac{p}{\theta_{1} a_{*}}\right)^{\prime}}}\left(f_{Q} v(y)^{\theta_{3} q} d y\right)^{\frac{1}{\theta_{3} q}} .
\end{aligned}
$$

By Lemma 3, we have $\theta_{1}\left(\frac{p}{\theta_{1} a_{*}}\right)^{\prime} \leq\left(\frac{p}{a}\right)^{\prime}$. By Hölder's inequality, we have

$$
\begin{aligned}
\tilde{M}_{\alpha, \theta_{1}, \theta_{3} q}\left(f^{\theta_{1}}, v\right)(x) \leq & C \sup _{Q \ni x}\left(l(Q)^{\alpha \cdot \frac{p}{a_{*}}} m_{3 Q}\left(|f w|^{\frac{p}{a_{*}}}\right)\right)^{\frac{a_{*}}{p}}\left(\frac{|3 Q|}{|Q|}\right)^{\frac{1}{a q_{0}}}|3 Q|^{-\frac{1}{r_{0}}} \\
& \times\left(\frac{|Q|}{|3 Q|}\right)^{\frac{1}{a q_{0}}}|3 Q|^{\frac{1}{r_{0}}}\left(f_{Q} v(y)^{\theta_{3} q} d y\right)^{\frac{1}{\theta_{3} q}}\left(f_{3 Q} w(y)^{-(p / a)^{\prime}} d y\right)^{\frac{1}{(p / a)^{\prime}}} .
\end{aligned}
$$

By the condition (2), we obtain

$$
\begin{aligned}
\tilde{M}_{\alpha, \theta_{1}, \theta_{3} q}\left(f^{\theta_{1}}, v\right)(x) & \leq C[v, w]_{a q_{0}, r_{0}, a q, p / a} \sup _{Q \ni x}\left(l(Q)^{\alpha \cdot \frac{p}{a_{*}}-\frac{n}{r_{0}} \cdot \frac{p}{a_{*}}} m_{3 Q}\left(|f w|^{\frac{p}{a_{*}}}\right)\right)^{\frac{a_{*}}{p}} \\
& =C[v, w]_{a q_{0}, r_{0}, a q, p / a} \cdot M_{\left(\alpha-\frac{n}{r_{0}}\right) \frac{p}{a_{*}}}\left(|f w|^{\frac{p}{a_{*}}}\right)(x)^{\frac{a_{*}}{p}} .
\end{aligned}
$$

This implies that

$$
\left|Q_{0}\right|^{\frac{1}{q_{0}}}\left(f_{Q_{0}} \tilde{M}_{\alpha, \theta_{1}, \theta_{3} q}\left(f^{\theta_{1}}, v\right)(x)^{q} d x\right)^{\frac{1}{q}} \leq C[v, w]_{a q_{0}, r_{0}, a q, p l a}\left\|M_{\left(\alpha-\frac{n}{r_{0}}\right) \frac{p}{a_{*}}}\left(|f w|^{\frac{p}{a_{*}}}\right)\right\|_{\mathcal{M}_{\frac{\mathcal{L}_{*}}{p}}^{\frac{a_{*}}{p}}}^{\frac{a_{*} q_{0}}{a_{*}}} .
$$

Since

$$
\frac{1}{q_{0}} \cdot \frac{p}{a_{*}}=\frac{1}{p_{0}} \cdot \frac{p}{a_{*}}-\frac{\left(\alpha-\frac{n}{r_{0}}\right) \cdot \frac{p}{a_{*}}}{n} \text { and } \quad \frac{\frac{a_{*} p_{0}}{p}}{\frac{p}{a_{*} q_{0}}}=\frac{a_{*}}{\frac{a_{*} q}{p}}
$$


lido Journal of Inequalities and Applications <wide>(2016) 2016:4

Page 18 of 23

by Theorem A, we have

$$
\left\|M_{\left(\alpha-\frac{n}{r_{0}}\right) \frac{p}{a_{*}}}\left(|f w|^{\frac{p}{a_{*}}}\right)\right\|_{\mathcal{M}_{\frac{\mathcal{M}_{*}}{p}}^{\frac{a_{*} q_{0}}{p}}}^{\frac{a_{*}}{p}} \leq\left\||f w|^{\frac{p}{a_{*}}}\right\|_{\mathcal{M}_{a_{*}}^{\frac{a_{*}}{p}}}^{\frac{a_{*}}{p}}=\|f w\|_{\mathcal{M}_{p}^{p_{0}}} .
$$

Therefore we have

$$
\|I I \cdot v\|_{\mathcal{M}_{q}^{q_{0}}} \leq C[v, w]_{a q_{0}, r_{0}, a q, p / a}\|f w\|_{\mathcal{M}_{p}^{p_{0}}}
$$

4.2 The estimate of $B$

Since $|b(x)-b(y)|^{m} \leq 2^{m-1}\left(\left|b(x)-m_{Q}(b)\right|^{m}+\left|m_{Q}(b)-b(y)\right|^{m}\right)$, we have

$$
\begin{aligned}
& \int_{3 Q}|b(x)-b(y)|^{m}|f(y)| d y \\
& \quad \leq C \int_{3 Q}\left|b(x)-m_{Q}(b)\right|^{m}|f(y)| d y+C \int_{3 Q}\left|m_{Q}(b)-b(y)\right|^{m}|f(y)| d y .
\end{aligned}
$$

Therefore we obtain

$$
\begin{aligned}
v(x) B \leq & C v(x) \sum_{\substack{Q \supset \mathcal{Q} Q_{0}, Q \in \mathcal{D}\left(\mathbb{R}^{n}\right)}}|Q|^{\left(\frac{\alpha}{n}-1\right)} \chi_{Q}(x) \int_{3 Q}\left|b(x)-m_{Q}(b)\right|^{m}|f(y)| d y \\
& +C v(x) \sum_{\substack{Q \supset Q_{0}, Q \in \mathcal{D}\left(\mathbb{R}^{n}\right)}}|Q|^{\left(\frac{\alpha}{n}-1\right)} \chi_{Q}(x) \int_{3 Q}\left|m_{Q}(b)-b(y)\right|^{m}|f(y)| d y \\
= & : C C_{1}[f, v](x)+C C_{2}[f, v](x) .
\end{aligned}
$$

By Hölder's inequality and the definition of the Morrey norm we obtain

$$
\begin{aligned}
C_{1}[f, v](x)= & v(x) \sum_{\substack{Q \supset \mathcal{Q} Q_{0}, Q \in \mathcal{D}\left(\mathbb{R}^{n}\right)}}|Q|^{\left(\frac{\alpha}{n}-1\right)} \chi_{Q}(x)\left|b(x)-m_{Q}(b)\right|^{m} \int_{3 Q}|f(y)| d y \\
\leq & v(x) \sum_{\substack{Q \supset Q_{0}, Q \in \mathcal{D}\left(\mathbb{R}^{n}\right)}}|Q|^{\left(\frac{\alpha}{n}-1\right)} \chi_{Q}(x)\left|b(x)-m_{Q}(b)\right|^{m}|3 Q|^{\frac{1}{p_{0}}}\left(f_{3 Q}|f(y)|^{p} w(y)^{p} d y\right)^{\frac{1}{p}} \\
& \times|3 Q|^{1-\frac{1}{p_{0}}}\left(f_{3 Q} w(y)^{-p^{\prime}} d y\right)^{\frac{1}{p^{\prime}}} \\
\leq & C\|f w\|_{\mathcal{M}_{p}^{p_{0}}} v(x) \sum_{\substack{Q \supset Q_{0}, Q \in \mathcal{D}\left(\mathbb{R}^{n}\right)}}|3 Q|^{\frac{\alpha}{n}-\frac{1}{p_{0}}}\left(f_{3 Q} w(y)^{-p^{\prime}} d y\right)^{\frac{1}{p^{\prime}}}\left|b(x)-m_{Q}(b)\right|^{m} .
\end{aligned}
$$

Since $\frac{1}{q_{0}}=\frac{1}{p_{0}}+\frac{1}{r_{0}}-\frac{\alpha}{n}$, the integral of $C_{1}[f, v](x)^{q}$ on $Q_{0}$ is evaluated as follows:

$$
\begin{aligned}
& \left|Q_{0}\right|^{\frac{1}{q_{0}}}\left(f_{Q_{0}} C_{1}[f, v](x)^{q} d x\right)^{\frac{1}{q}} \\
& \leq C\|f w\|_{\mathcal{M}_{p}^{p_{0}}} \sum_{\substack{Q \supset Q_{0}, Q \in \mathcal{D}\left(\mathbb{R}^{n}\right)}}\left|Q_{0}\right|^{\frac{1}{q_{0}}}|3 Q|^{\frac{1}{r_{0}}-\frac{1}{q_{0}}}\left(f_{3 Q} w(y)^{-p^{\prime}} d y\right)^{\frac{1}{p^{\prime}}}
\end{aligned}
$$




$$
\begin{aligned}
& \times\left(f_{Q_{0}} v(x)^{q}\left|b(x)-m_{Q}(b)\right|^{m q} d x\right)^{\frac{1}{q}} \\
= & C\|f w\|_{\mathcal{M}_{p}^{p_{0}}} \sum_{\substack{Q \supset Q_{0}, Q \in \mathcal{D}\left(\mathbb{R}^{n}\right)}}\left(\frac{\left|Q_{0}\right|}{|3 Q|}\right)^{\frac{1}{q_{0}}}|3 Q|^{\frac{1}{r_{0}}}\left(f_{3 Q} w(y)^{-p^{\prime}} d y\right)^{\frac{1}{p^{\prime}}} \\
& \times\left(f_{Q_{0}} v(x)^{q}\left|b(x)-m_{Q}(b)\right|^{m q} d x\right)^{\frac{1}{q}} .
\end{aligned}
$$

By Hölder's inequality for $\theta_{4}>1$ as in Lemma 3, we have

$$
\begin{aligned}
& \left(f_{Q_{0}} v(x)^{q}\left|b(x)-m_{Q}(b)\right|^{m q} d x\right)^{\frac{1}{q}} \\
& \quad \leq\left(f_{Q_{0}} v(x)^{q \theta_{4}} d x\right)^{\frac{1}{q \theta_{4}}}\left(f_{Q_{0}}\left|b(x)-m_{Q}(b)\right|^{m q \theta_{4}^{\prime}} d x\right)^{\frac{1}{q \theta_{4}^{\prime}}} .
\end{aligned}
$$

We evaluate $\left|b(x)-m_{Q}(b)\right|$. If $Q \supsetneqq Q_{0}$ and $Q \in \mathcal{D}\left(\mathbb{R}^{n}\right)$, then there exists $k=1,2, \ldots$, such that $Q_{k}:=Q, Q_{j} \in \mathcal{D}\left(\mathbb{R}^{n}\right), Q_{j} \supsetneqq Q_{j-1}$ and $\left|Q_{j}\right|=2^{n}\left|Q_{j-1}\right|(j=1,2, \ldots, k)$. By the triangle inequality, we obtain

$$
\begin{aligned}
\left|b(x)-m_{Q}(b)\right| & \leq\left|b(x)-m_{Q_{0}}(b)\right|+\left|m_{Q_{0}}(b)-m_{Q}(b)\right| \\
& =\left|b(x)-m_{Q_{0}}(b)\right|+\left|\sum_{j=1}^{k}\left(m_{Q_{j-1}}(b)-m_{Q_{j}}(b)\right)\right| \\
& \leq\left|b(x)-m_{Q_{0}}(b)\right|+\sum_{j=1}^{k}\left|m_{Q_{j-1}}(b)-m_{Q_{j}}(b)\right| .
\end{aligned}
$$

Moreover, we have

$$
\begin{aligned}
\left|m_{Q_{j-1}}(b)-m_{Q_{j}}(b)\right| & =\left|f_{Q_{j-1}} b(y) d y-m_{Q_{j}}(b)\right| \\
& =\left|f_{Q_{j-1}}\left(b(y)-m_{Q_{j}}(b)\right) d y\right| \\
& \leq f_{Q_{j-1}}\left|b(y)-m_{Q_{j}}(b)\right| d y \\
& \leq \frac{2^{n}}{\left|Q_{j}\right|} \int_{Q_{j}}\left|b(y)-m_{Q_{j}}(b)\right| d y \\
& \leq 2^{n}\|b\|_{\text {Вмо }} \quad(j=1,2, \ldots),
\end{aligned}
$$

where we invoke Definition 2 for the last line. By the inequality $(a+b)^{m} \leq 2^{m-1}\left(a^{m}+b^{m}\right)$ :

$$
\begin{aligned}
\left|b(x)-m_{Q}(b)\right|^{m} & \leq\left(\left|b(x)-m_{\mathrm{Q}_{0}}(b)\right|+2^{n} k\|b\|_{\mathrm{BMO}}\right)^{m} \\
& \leq C\left(\left|b(x)-m_{\mathrm{Q}_{0}}(b)\right|^{m}+2^{m n} k^{m}\|b\|_{\mathrm{BMO}}^{m}\right) .
\end{aligned}
$$


lido Journal of Inequalities and Applications <wide>(2016<wide>) 2016:4

Page 20 of 23

By the estimates (6), (7), and Hölder's inequality for $(p / a)^{\prime}>p^{\prime}$, we obtain

$$
\begin{aligned}
& \sum_{\substack{Q \supset Q_{0}, Q \in \mathcal{D}\left(\mathbb{R}^{n}\right)}}\left(\frac{\left|Q_{0}\right|}{|3 Q|}\right)^{\frac{1}{q_{0}}}|3 Q|^{\frac{1}{r_{0}}}\left(f_{3 Q} w(y)^{-p^{\prime}} d y\right)^{\frac{1}{p^{\prime}}}\left(f_{Q_{0}} v(x)^{q}\left|b(x)-m_{Q}(b)\right|^{m q} d x\right)^{\frac{1}{q}} \\
& \leq C \sum_{k=1}^{\infty} \sum_{\substack{Q_{Q_{k}} \in \mathcal{D}\left(\mathbb{R}^{n}\right), Q_{k} \supset Q_{0},\left|Q_{k}\right| 2^{k n}\left|Q_{0}\right|}}\left(\frac{\left|Q_{0}\right|}{\left|3 Q_{k}\right|}\right)^{\frac{1}{q_{0}}}\left|3 Q_{k}\right|^{\frac{1}{r_{0}}}\left(f_{3 Q_{k}} w(y)^{-(p / a)^{\prime}} d y\right)^{\frac{1}{(p / a)^{\prime}}} \\
& \quad \times\left(f_{Q_{0}} v(x)^{q \theta_{4}} d x\right)^{\frac{1}{\theta_{4}}}\left(f_{Q_{0}}\left(\left|b(x)-m_{Q_{0}}(b)\right|^{m}+2^{m n} k^{m}\|b\|_{\mathrm{BMO}}^{m}\right)^{q \theta_{4}^{\prime}} d x\right)^{\frac{1}{\theta_{4}^{\prime}}} .
\end{aligned}
$$

By the triangle inequality on $L^{q \theta_{4}^{\prime}}\left(\mathbb{R}^{n}\right)$, we obtain

$$
\begin{aligned}
& \left(f_{\mathrm{Q}_{0}}\left(\left|b(x)-m_{\mathrm{Q}_{0}}(b)\right|^{m}+2^{m n} k^{m}\|b\|_{\mathrm{BMO}}^{m}\right)^{q \theta_{4}^{\prime}} d x\right)^{\frac{1}{q \theta_{4}^{\prime}}} \\
& \leq\left(f_{\mathrm{Q}_{0}}\left|b(x)-m_{\mathrm{Q}_{0}}(b)\right|^{m q \theta_{4}^{\prime}} d x\right)^{\frac{1}{q \theta_{4}^{\prime}}}+\left(f_{\mathrm{Q}_{0}}\left(2^{m n} k^{m}\|b\|_{\mathrm{BMO}}^{m}\right)^{q \theta_{4}^{\prime}} d x\right)^{\frac{1}{q \theta_{4}^{\prime}}} .
\end{aligned}
$$

By the estimate (8), we obtain

$$
\begin{aligned}
\sum_{k=1}^{\infty} & \sum_{\substack{Q_{k} \in \mathcal{D}\left(\mathbb{R}^{n}\right), Q_{k} \supset Q_{0},\left|Q_{k}\right|=2^{k n}\left|Q_{0}\right|}}\left(\frac{\left|Q_{0}\right|}{\left|3 Q_{k}\right|}\right)^{\frac{1}{q_{0}}}\left|3 Q_{k}\right|^{\frac{1}{r_{0}}}\left(f_{3 Q_{k}} w(y)^{-(p / a)^{\prime}} d y\right)^{\frac{1}{(p / a)^{\prime}}}\left(f_{Q_{0}} v(x)^{q \theta_{4}} d x\right)^{\frac{1}{\theta_{4}}} \\
& \times\left(f_{Q_{0}}\left(\left|b(x)-m_{Q_{0}}(b)\right|^{m}+2^{m n} k^{m}\|b\|_{\mathrm{BMO}}^{m}\right)^{q \theta_{4}^{\prime}} d x\right)^{\frac{1}{q \theta_{4}^{\prime}}} \\
\leq & \sum_{k=1}^{\infty} \sum_{Q_{k} \supset Q_{0},\left|Q_{k}\right|=2^{k n}\left|Q_{0}\right|}\left(\frac{\left|Q_{0}\right|}{\left|3 Q_{k}\right|}\right)^{\frac{1}{q_{0}}}\left|3 Q_{k}\right|^{\frac{1}{r_{0}}} \\
& \times\left(f_{3 Q_{k}} w(y)^{-(p / a)^{\prime}} d y\right)^{\frac{1}{(p / a)^{\prime}}}\left(f_{Q_{0}} v(x)^{q \theta_{4}} d x\right)^{\frac{1}{q \theta_{4}}} \\
& \times\left\{\left(f_{Q_{0}}\left|b(x)-m_{Q_{0}}(b)\right|^{m q \theta_{4}^{\prime}} d x\right)^{\frac{1}{q \theta_{4}^{\prime}}}+\left(f_{Q_{0}}\left(2^{m n} k^{m}\|b\|_{\mathrm{BMO}}^{m}\right)^{q \theta_{4}^{\prime}} d x\right)^{\frac{1}{q_{4}^{\prime}}}\right\} .
\end{aligned}
$$

By Lemma 1, we have

$$
\left(f_{\mathrm{Q}_{0}}\left|b(x)-m_{\mathrm{Q}_{0}}(b)\right|^{m q \theta_{4}^{\prime}} d x\right)^{\frac{1}{q \theta_{4}^{\prime}}} \leq C\|b\|_{\mathrm{BMO}}^{m} .
$$

The estimate (9) gives us the following:

$$
\begin{aligned}
& \left(f_{\mathrm{Q}_{0}}\left|b(x)-m_{\mathrm{Q}_{0}}(b)\right|^{m q \theta_{4}^{\prime}} d x\right)^{\frac{1}{\theta_{4}^{\prime}}}+\left(f_{\mathrm{Q}_{0}}\left(2^{m n} k^{m}\|b\|_{\mathrm{BMO}}^{m}\right)^{q \theta_{4}^{\prime}} d x\right)^{\frac{1}{q \theta_{4}^{\prime}}} \\
& \quad \leq C\|b\|_{\mathrm{BMO}}^{m}\left(1+2^{m n} k^{m}\right) .
\end{aligned}
$$


As a consequence of (10), we obtain the following inequality:

$$
\begin{aligned}
& \sum_{\substack{Q \mathcal{Q}_{Q} Q_{0}, Q \in \mathcal{D}\left(\mathbb{R}^{n}\right)}}\left(\frac{\left|Q_{0}\right|}{|3 Q|}\right)^{\frac{1}{q_{0}}}|3 Q|^{\frac{1}{r_{0}}}\left(f_{3 Q} w(y)^{-p^{\prime}} d y\right)^{\frac{1}{p^{\prime}}}\left(f_{Q_{0}} v(x)^{q}\left|b(x)-m_{Q}(b)\right|^{m q} d x\right)^{\frac{1}{q}} \\
& \leq C\|b\|_{\mathrm{BMO}}^{m} \sum_{k=1}^{\infty} \sum_{\substack{Q_{k} \in \mathcal{D}\left(\mathbb{R}^{n}\right), Q_{k} \supset Q_{0},\left|Q_{k}\right|=2^{k n}\left|Q_{0}\right|}}\left(\frac{\left|Q_{0}\right|}{\left|3 Q_{k}\right|}\right)^{\frac{1}{a q_{0}}}\left|3 Q_{k}\right|^{\frac{1}{r_{0}}}\left(f_{3 Q_{k}} w(y)^{-(p / a)^{\prime}} d y\right)^{\frac{1}{(p / a)^{\prime}}} \\
& \quad \times\left(f_{Q_{0}} v(x)^{q \theta_{4}} d x\right)^{\frac{1}{q \theta_{4}}}\left(1+2^{m n} k^{m}\right)\left(\frac{\left|Q_{0}\right|}{\left|3 Q_{k}\right|}\right)^{\frac{1}{q_{0}}\left(1-\frac{1}{a}\right)} .
\end{aligned}
$$

By the condition (2), we have

$$
\begin{aligned}
& \sum_{\substack{Q \supset Q_{0}, Q \in \mathcal{D}\left(\mathbb{R}^{n}\right)}}\left(\frac{\left|Q_{0}\right|}{|3 Q|}\right)^{\frac{1}{q_{0}}}|3 Q|^{\frac{1}{r_{0}}}\left(f_{3 Q} w(y)^{-p^{\prime}} d y\right)^{\frac{1}{p^{\prime}}}\left(f_{Q_{0}} v(x)^{q}\left|b(x)-m_{Q}(b)\right|^{m q} d x\right)^{\frac{1}{q}} \\
& \leq C\|b\|_{\mathrm{BMO}}^{m}[v, w]_{a q_{0}, r_{0}, a q, p / a} \sum_{k=1}^{\infty} \sum_{\substack{Q_{k} \in \mathcal{D}\left(\mathbb{R}^{n}\right), Q_{k} \supset Q_{0},\left|Q_{k}\right|=2^{k n}\left|Q_{0}\right|}}\left(1+2^{m n} k^{m}\right) 2^{-\frac{k n}{q_{0}}\left(1-\frac{1}{a}\right)} \\
& \quad=C\|b\|_{\mathrm{BMO}}^{m}[v, w]_{a q_{0}, r_{0}, a q, p / a} \sum_{k=1}^{\infty}\left(1+2^{m n} k^{m}\right) 2^{-\frac{k n}{q_{0}}\left(1-\frac{1}{a}\right)} \\
& \leq C\|b\|_{\mathrm{BMO}}^{m}[v, w]_{a q_{0}, r_{0}, a q, p / a .}
\end{aligned}
$$

Therefore we obtain

$$
\left\|C_{1}[f, v]\right\|_{\mathcal{M}_{q}^{q_{0}}} \leq C[v, w]_{a q_{0}, r_{0}, a q, p / a}\|b\|_{\mathrm{BMO}}^{m}\|f w\|_{\mathcal{M}_{p}^{p_{0}}} .
$$

Next, we evaluate $C_{2}[f, v](x)$. By Hölder's inequality for $\theta_{5} \in(1, p)$ in Lemma 3 , we have

$$
\begin{aligned}
C_{2}[f, v](x)= & v(x) \sum_{\substack{Q \supset \mathcal{Q} Q_{0}, Q \in \mathcal{D}\left(\mathbb{R}^{n}\right)}} l(Q)^{\alpha-n} \chi_{Q}(x)\left(\int_{3 Q}\left|m_{Q}(b)-b(y)\right|^{m} f(y) d y\right) \\
\leq & v(x) \sum_{\substack{Q \supset Q_{0}, Q \in \mathcal{D}\left(\mathbb{R}^{n}\right)}} l(Q)^{\alpha-n} \chi_{Q}(x)\left(\int_{3 Q}\left|m_{Q}(b)-b(y)\right|^{m \theta_{5}^{\prime}} d y\right)^{\frac{1}{\theta_{5}^{\prime}}} \\
& \times\left(\int_{3 Q}|f(y)|^{\theta_{5}} d y\right)^{\frac{1}{\theta_{5}}} .
\end{aligned}
$$

By Hölder's inequality for $\frac{p}{\theta_{5}}>1$, we obtain

$$
\begin{aligned}
C_{2}[f, v](x) \leq & v(x) \sum_{\substack{Q \supset Q_{0}, Q \in \mathcal{D}\left(\mathbb{R}^{n}\right)}} l(Q)^{\alpha-\frac{n}{p_{0}}} \chi_{Q}(x)\left(f_{3 Q}\left|m_{Q}(b)-b(y)\right|^{m \theta_{5}^{\prime}} d y\right)^{\frac{1}{\theta_{5}^{\prime}}}|3 Q|^{\frac{1}{p_{0}}} \\
& \times\left(f_{3 Q} w(y)^{-\theta_{5}\left(\frac{p}{\theta_{5}}\right)^{\prime}} d y\right)^{\frac{1}{\theta_{5}\left(\frac{p}{\theta_{5}}\right)^{\prime}}}\left(f_{3 Q}|f(y)|^{p} w(y)^{p} d y\right)^{\frac{1}{p}}
\end{aligned}
$$


Taking the Morrey norm, we obtain

$$
\begin{aligned}
C_{2}[f, v](x) \leq & \|f w\|_{\mathcal{M}_{p}^{p_{0}} v(x)} \sum_{\substack{Q \mathcal{P} Q_{0}, Q \in \mathcal{D}\left(\mathbb{R}^{n}\right)}} l(Q)^{\alpha-\frac{n}{p_{0}}} \chi_{Q}(x)\left(f_{3 Q}\left|m_{Q}(b)-b(y)\right|^{m \theta_{5}^{\prime}} d y\right)^{\frac{1}{\theta_{5}^{\prime}}} \\
& \times\left(f_{3 Q} w(y)^{-\theta_{5}\left(\frac{p}{\theta_{5}}\right)^{\prime}} d y\right)^{\frac{1}{\theta_{5}\left(\frac{p}{\theta_{5}}\right)^{\prime}}} .
\end{aligned}
$$

Using Lemma 1, we have

$$
C_{2}[f, v](x) \leq\|b\|_{\mathrm{BMO}}^{m}\|f w\|_{\mathcal{M}_{p}^{p_{0}}} v(x) \sum_{\substack{Q \supset Q_{0}, Q \in \mathcal{D}\left(\mathbb{R}^{n}\right)}} l(Q)^{\alpha-\frac{n}{p_{0}}} \chi_{Q}(x)\left(f_{3 Q} w(y)^{-\theta_{5}\left(\frac{p}{\theta_{5}}\right)^{\prime}} d y\right)^{\frac{1}{\theta_{5}\left(\frac{p}{\left.\theta_{5}\right)^{\prime}}\right.}} .
$$

Since we have the assumption that $a \geqq \frac{p}{\left(\theta_{5}\left(\frac{p}{\theta_{5}}\right)^{\prime}\right)^{\prime}}>1$, using Hölder's inequality, we obtain

$$
C_{2}[f, v](x) \leq C\|b\|_{\mathrm{BMO}}^{m}\|f w\|_{\mathcal{M}_{p}^{p_{0}}} v(x) \sum_{\substack{Q \supset Q_{0}, Q \in \mathcal{D}\left(\mathbb{R}^{n}\right)}} l(Q)^{\alpha-\frac{n}{p_{0}}} \chi_{Q}(x)\left(f_{3 Q} w(y)^{-(p / a)^{\prime}} d y\right)^{\frac{1}{(p / a)^{\prime}}} .
$$

The integral of $C_{2}[f, v](x)^{q}$ on $Q_{0}$ is evaluated as follows:

$$
\begin{aligned}
\left|Q_{0}\right|^{\frac{1}{q_{0}}}\left(f_{Q_{0}} C_{2}[f, v](x)^{q} d x\right)^{\frac{1}{q}} \\
\leq C\|b\|_{\mathrm{BMO}}^{m}\|f w\|_{\mathcal{M}_{p}^{p_{0}}} \sum_{\substack{Q \mathcal{D}_{0}, Q \in \mathcal{D}\left(\mathbb{R}^{n}\right)}} l(Q)^{\alpha-\frac{n}{p_{0}}}\left|Q_{0}\right|^{\frac{1}{q_{0}}} \\
\quad \times\left(f_{Q_{0}} v(x)^{q} d x\right)^{\frac{1}{q}}\left(f_{3 Q} w(y)^{-(p / a)^{\prime}} d y\right)^{\frac{1}{(p / a)^{\prime}}} \\
\leq C\|b\|_{\mathrm{BMO}}^{m}\|f w\|_{\mathcal{M}_{p}^{p_{0}}} \sum_{\substack{Q \mathcal{Z}_{0}, Q \in \mathcal{D}\left(\mathbb{R}^{n}\right)}}\left(\frac{\left|Q_{0}\right|}{|3 Q|}\right)^{\frac{1}{a q_{0}}}|3 Q|^{\frac{1}{r_{0}}} \\
\quad \times\left(f_{Q_{0}} v(x)^{a q} d x\right)^{\frac{1}{a_{q}}}\left(f_{3 Q} w(y)^{-(p / a)^{\prime}} d y\right)^{\frac{1}{(p / a)^{\prime}}}\left(\frac{\left|Q_{0}\right|}{|3 Q|}\right)^{\frac{1}{q_{0}}\left(1-\frac{1}{a}\right)} .
\end{aligned}
$$

By the condition (2), we have

$$
\begin{aligned}
& \left|Q_{0}\right|^{\frac{1}{q_{0}}}\left(f_{Q_{0}} C_{2}[f, v](x)^{q} d x\right)^{\frac{1}{q}} \\
& \quad \leq C[v, w]_{a q_{0}, r_{0}, a q, p / a}\|b\|_{\mathrm{BMO}}^{m}\|f w\|_{\mathcal{M}_{p}^{p_{0}}} \sum_{\substack{Q \supset \mathcal{Q}_{0}, Q \in \mathcal{D}\left(\mathbb{R}^{n}\right)}}\left(\frac{\left|Q_{0}\right|}{|3 Q|}\right)^{\frac{1}{q_{0}}\left(1-\frac{1}{a}\right)} \\
& \quad \leq C[v, w]_{a q_{0}, r_{0}, a q, p / a}\|b\|_{\mathrm{BMO}}^{m}\|f w\|_{\mathcal{M}_{p}^{p_{0}}} .
\end{aligned}
$$

We obtain the desired result. 


\section{Competing interests}

The author declares that they have no competing interests.

\section{Acknowledgements}

The author wish to thank Professor Y Sawano for a rich lecture of higher order commutators generated by BMO-functions and the fractional integral operator.

Received: 16 September 2015 Accepted: 21 December 2015 Published online: 04 January 2016

\section{References}

1. Iida, T, Sato, E, Sawano, Y, Tanaka, H: Weighted norm inequalities for multilinear fractional operators on Morrey spaces. Stud. Math. 205, 139-170 (2011)

2. Sawano, Y, Sugano, S, Tanaka, H: A bilinear estimate for commutators of fractional integral operators. In: Potential Theory and Its Related Fields. RIMS Kôkyûroku Bessatsu, vol. B43, pp. 155-170. Res. Inst. Math. Sci., Kyoto (2013)

3. Grafakos, L: Modern Fourier Analysis, 2nd edn. Graduate Texts in Math., vol. 250. Springer, New York (2008)

4. Lu, S, Ding, Y, Yan, D: Singular Integrals and Related Topics. World Scientific, Singapore (2007)

5. Adams, D: A note on Riesz potentials. Duke Math. J. 42, 765-778 (1975)

6. Di-Fazio, G, Ragusa, MA: Commutators and Morrey spaces. Boll. UMI 7, 323-332 (1991)

7. Komori, Y, Mizuhara, T: Notes on commutators and Morrey spaces. Hokkaido Math. J. 32, 345-353 (2003)

8. lida, T, Sawano, Y, Tanaka, H: Atomic decomposition for Morrey spaces. Z. Anal. Anwend. 33, 149-170 (2014)

9. Sawano, Y, Sugano, S, Tanaka, H: Generalized fractional integral operators and fractional maximal operators in the framework of Morrey spaces. Trans. Am. Math. Soc. 363, 6481-6503 (2011)

10. Ding, Y, Lu, S: Weighted norm inequalities for fractional integral operators with rough kernel. Can. J. Math. 50, 29-39 (1998)

11. Ding, Y, Lu, S: Higher order commutators for a class of rough operators. Ark. Mat. 37, 33-44 (1999)

12. lida, T: Weighted inequalities on Morrey spaces for linear and multilinear fractional integrals with homogeneous kernels. Taiwan. J. Math. 18, 147-185 (2014)

13. Duoandikoetxea, J: Fourier Analysis. Grad. Studies in Math., vol. 29. Am. Math. Soc., Providence (2001)

14. Pérez, C: Two weighted inequalities for potential and fractional type maximal operators. Indiana Univ. Math. J. 43, 663-683 (1994)

15. Pérez, C: On sufficient conditions for the boundedness of the Hardy-Littlewood maximal operator between $L^{p}$ spaces with different weights. Proc. Lond. Math. Soc. (3) 71, 135-157 (1995)

16. Pérez, C: Sharp $L^{p}$-weighted Sobolev inequalities. Ann. Inst. Fourier (Grenoble) 45, 809-824 (1995)

\section{Submit your manuscript to a SpringerOpen ${ }^{\circ}$ journal and benefit from:}

- Convenient online submission

Rigorous peer review

- Immediate publication on acceptance

- Open access: articles freely available online

- High visibility within the field

- Retaining the copyright to your article 